\title{
How Do We Know When a Lymph Node Dissection is Adequate?
}

\author{
Vernon K. Sondak, MD ${ }^{1,2}$ \\ ${ }^{1}$ Department of Cutaneous Oncology, Moffitt Cancer Center, Tampa, FL; ${ }^{2}$ Departments of Oncologic Sciences and \\ Surgery, University of South Florida College of Medicine, Tampa, FL
}

How do we know when a lymph node dissection is adequate? Like so many questions, the answer depends in part on who is asking. For our melanoma patients, the answer is pragmatic. Their node dissection is adequate if it fulfills the primary goals of the procedure of providing life-long regional disease control and accurate staging informationideally with the lowest possible morbidity. Of course, this "answer" for an individual patient cannot readily be determined early on after surgery; only time will tell. For melanoma surgeons, as well as for our oncology colleagues evaluating patients for clinical trial eligibility, there are occasions when we must assess whether a lymph node dissection is adequate during the early postoperative period. Some adjuvant therapy clinical trials mandate a minimum number of nodes be removed, although this number is rarely evidence-based. A scenario surgical oncologists confront with some frequency is the need to assess the adequacy of a node dissection that yielded only a relatively small number of pathologically examined lymph nodes.

Possible explanations: the node dissection was adequate and there were simply few lymph nodes in that patient; the dissection was adequate but the pathologic evaluation did not identify or enumerate every harvested node (most of which were likely small and tumor-free); or an inadequate node dissection was performed, leaving lymph nodes behind that ought to have been removed and raising the specter of an increased risk of regional recurrence and potentially underestimating the patient's risk of distant metastasis. Increasingly, others besides the patient and the surgeon or oncologist are asking about the adequacy of surgery: quality assurance evaluations have become a fact of life for most clinicians, and an appropriate goal of these

(C) Society of Surgical Oncology 2011

Published Online: 3 May 2011

V. K. Sondak, MD

e-mail: vernon.sondak@moffitt.org evaluations should be to identify surgeons who consistently fail to perform adequate surgeries and correct this through remedial education and mentoring or, failing that, restricted privileges. Finally, it is increasingly easy to envision a future where reimbursement for individual surgical procedures is tied to fulfillment of specific criteria that demonstrate its "adequacy." Will we soon see reimbursement for lymph node dissections tied to removal of a minimum number of lymph nodes?

In this issue of the Annals of Surgical Oncology, Spillane et al. provide additional information about the relationship of lymph node retrieval numbers and the adequacy of the procedure. ${ }^{1}$ Taken together with this team's other publications, ${ }^{2-4}$ generated from the large dataset of the Melanoma Institute Australia, a picture emerges of minimum node numbers retrieved from at least $90 \%$ of patients who undergo lymphadenectomies by experienced melanoma surgeons: 20 neck nodes if at least four levels are dissected but only 6 after three-level dissection, and 10 axillary nodes-but surprisingly only 7 nodes from the groin. The 90th percentile figures are of more practical value than mean or median numbers, but further validation from around the world - and ideally even our own individual institutions-would be of great benefit. Prospective data from the two Multicenter Selective Lymphadenectomy Trials will be particularly informative, because these protocols define "required" number of lymph nodes for adequate lymphadenectomies that in some cases are higher than the Australians' 90th percentile values (Morton DL, personal communication).

Beyond the number of lymph nodes indicated on the pathology report, how can we tell if a patient has had an adequate regional node dissection? Regardless of why the issue arises, we have developed a standard algorithm for clinically assessing node dissection adequacy and making decisions regarding when to recommend redo node dissection. 
The first step is to query the pathologist about the node dissection specimen itself. Reexamination of the gross specimen should be requested, with submission of additional tissue, potentially increasing the yield of small nodes missed on initial pathologic evaluation. In fact, the pathologist often can tell whether the tissue sampling has been inadequate by assessing the size range of the nodes on the microscopic slides: the absence of any small (2-3 mm) lymph nodes may be an indication that further tissue sampling is likely to yield additional nodes. In this regard, it is relevant to note that the standard method of gross dissection in most pathology laboratories is to inspect visually and palpate for gross identification of lymph nodes within node dissection specimens. Detection of lymph nodes often is enhanced by a period of formalin fixation. Visualizing and/or palpating lymph nodes can be more difficult if there has been extensive previous surgery, which likely is at least a partial explanation for the frequent observation that fewer nodes are found in lymphadenectomy specimens performed after an initial sentinel node biopsy as opposed to after fine needle aspiration of a palpable lymph node. A landmark study conducted in breast cancer axillary dissection specimens before the sentinel node era showed that axillary fat clearing markedly increased node yield but failed to identify additional positive nodes if the initially identified nodes were negative. ${ }^{5}$ More involved nodes were found if the initial nodes were positive, so whether this technique would help in the sentinel node era might bear reexamination.

Intuitively, one would think that reviewing the dictated operative note from the lymphadenectomy should allow a reliable assessment of the adequacy of the procedure, but in practice this is rarely the case. Remunerative considerations lead to many lesser procedures being labeled as "radical lymphadenectomies," and the dictation often seems aimed more at justifying the billing code than establishing the procedure's oncologic adequacy.

A focused history and physical examination can provide important clinical clues to the completeness of a lymphadenectomy. We begin with a series of specific questions: Was the procedure performed under general anesthesia? Was the patient admitted to the hospital postoperatively? Was a drain used, and if so for how long did it remain in place? Did the patient notice any paresthesias or numbness postoperatively? Admittedly, no single response can prove or disprove the completeness of the dissection, but in aggregate they can paint a compelling picture: it is the rare melanoma node dissection that can be oncologically complete yet performed under local anesthesia as an outpatient, with no drain and no numbness or paresthesias. Examination of the surgical site, noting the length and location of the incision and comparing the degree of soft tissue remaining to the contralateral side is the next step: a complete lymphadenectomy generally results in a very noticeable asymmetry of the soft tissues, with the potential exception of a groin dissection in which sartorius transposition has been performed. As a routine, the examination also includes assessment of the strength of the relevant muscles innervated by motor nerves within the basin: tongue and trapezius (hypoglossal and spinal accessory nerves) for the neck; scapular winging and latissimus (long thoracic and thoracodorsal nerves) for the axilla; and adductor and quadriceps (obturator and femoral nerves) for the groin. While this examination rarely sheds light on the completeness of the original operation, given the increased risk of nerve injury associated with reoperative surgery, it is prudent to document the preoperative nerve status whenever a redo lymphadenectomy is being contemplated.

Whereas routine imaging is rarely helpful in assessing the completeness of a node dissection, particularly if the indication for the dissection was microscopic disease, we have found nodal basin ultrasonography to be very helpful. If residual lymph nodes are seen, whether abnormal or not, this provides strong evidence to the surgeon and the patient that the dissection has been less than complete and helps justify and sometimes guide reoperation. If extensive postsurgical artifact hampers the ultrasound but no obvious nodes are seen, the scan can be repeated in several weeks and even if necessary in several months. When the adequacy of the dissection remains uncertain but reoperation is not undertaken, the basin also can be monitored with serial ultrasonography. At present, there is virtually no prospective data on how often ultrasound would identify residual nodes after standard "complete" radical lymphadenectomy in the neck, axilla, or groin, or how useful it will be in this surveillance role. Such studies would, however, be relatively straightforward to conduct and might provide valuable data. If the initial indication for the lymphadenectomy was macroscopic disease identifiable on preoperative imaging, repeat imaging-especially repeat PET/CT fusion scanning - after resolution of the immediate postsurgical changes also is routinely employed.

Even after this evaluation, the decision to perform redo lymphadenectomy is always a difficult one. Patients and their families are upset that they have undergone an apparently inadequate initial procedure, and all involved must recognize the increased morbidity associated with reoperation, although precise estimates of that morbidity are virtually impossible to provide from the available literature. Cases where the initial procedure was done through a nonstandard incision can be particularly problematic. Nonetheless, we have found that in carefully selected patients whose initial procedure was inadequate, repeat lymphadenectomy can be safely accomplished in the vast majority of cases and almost always leads to a substantial lymph node yield, and in our opinion this elective 
reoperation is safer and more successful than reoperation for recurrence in a partially dissected nodal basin. Although there is evidence that postoperative radiation can decrease regional recurrence, ${ }^{6}$ we have not been willing to rely on radiation alone if our assessment indicates the node dissection has been incomplete.

The mere fact that the clinical scenario described herein occurs as often as it does signifies a need for better education of general surgeons and even surgical oncologists about the principles and techniques of melanoma surgery, and supports the potential value of ongoing quality assurance and performance improvement efforts in surgery. Specialty surgical oncologists need to show leadership in our communities to improve overall outcomes, despite the very real challenges faced in confronting our fellow surgeons about their practice patterns-often based on relatively incomplete information. A generous dose of humility should come with each effort to judge our colleagues' performance from afar.

What about the potential that third-party payers will take note of the emerging data about lymph node yields and begin to deny reimbursement for cases reporting fewer nodes than a preset lower limit? Any such effort seems premature and troubling, as indicated by the clinical evaluation process described above, just relying on node number is not adequate to fully assess surgical adequacy. Even using the threshold values derived of Spillane et al., $10 \%$ of patient who undergo an adequate dissection by highly competent surgeons will yield subthreshold numbers of nodes, and in some of those cases the low number reflects less aggressive processing of the pathology specimen, not incomplete surgery. So while education and quality assurance monitoring are well justified based on the yield of nodes in lymphadenectomy specimens, at present reimbursement determinations are not. In the future, technology may offer a way to address this: in this era of built- in cell phone digital videocameras, we already have the technology to document quickly the adequacy of a lymphadenectomy. What we may lack is the infrastructure to incorporate such video evidence in our records, and an agreed upon format for readily verifying the completeness of each type of lymphadenectomy. Even more importantly, we lack an understanding of potential medicolegal implications such photodocumentation might have, although the experience with laparoscopic and endoscopic procedures that routinely provide an enduring video record should provide some precedent. Once again, as specialist surgical oncologists, the clear and present need is for leadership-in this case to preclude accountants and bureaucrats from telling us which cases are adequate for reimbursement and which are not. Our colleagues in Australia have provided a starting point; it is up to all of us to take the next steps.

\section{REFERENCES}

1. Spillane AJ, Haydu L, McMillan W, Stretch JR, Thompson JF. Quality assurance parameters and predictors of outcome for ilioinguinal and inguinal dissection in a contemporary melanoma patient population. Ann Surg Oncol. 2011, this issue.

2. Spillane AJ, Cheung BL, Stretch JR, et al. Proposed quality standards for regional lymph node dissections in patients with melanoma. Ann Surg. 2009;249:473-80.

3. Spillane AJ, Winstanley J, Thompson JF. Lymph node ratio in melanoma: a marker of variation in surgical quality? Cancer. 2009;115:2384-7.

4. Spillane AJ, Cheung BL, Winstanley J, Thompson JF. Lymph node ratio provides prognostic information in addition to AJCC N stage in patients with melanoma, even if quality of surgery is standardized. Ann Surg. 2011;253:109-15.

5. Morrow M, EvanS J, Rosen PP, Kinne DW. Does clearing of axillary lymph nodes contribute to accurate staging of breast carcinoma? Cancer. 1984;53:1329-32.

6. Rao NG, Yu H-HM, Trotti A III, Sondak VK. The role of radiation therapy in the management of cutaneous melanoma. Surg Oncol Clin North Am. 2011;20:115-31. 